\title{
Bubble Rise in a Packed Bed Saturated with
}

Liquids

ROBERT L. GORRING and DONALD L. KATZ

University of Michigan, Ann Arbor, Michigan

\begin{abstract}
Experimental data were determined for drag coefficients on fluid bubbles rising in a saturated packed bed and in clear liquids. Air and benzene were used to form bubbles in glycerine solution, water, and normal heptane. One-inch diameter glass spheres in hexagonal, random, and cubic packings constituted the bed. The range in Reynolds number was from 0.1 to 1,000 . It was found that the drag coefficients in the hexagonal and random arrays were from two to three times those in clear liquids, while the drag on bubbles rising in cubic packings was the same as that for clear liquids. The method of correlation involved the use of the minor diameter in the drag coefficient and the major diameter in the Reynolds number. This innovation improved the correlation for bubble rise in clear liquids.
\end{abstract}

The original motivation behind this investigation was directed toward determining the rate of rise of very small gas bubbles in porous media containing water and natural gas. When no data could be found in the literature, it was decided to investigate experimentally the general problem of bubble rise in packed beds. To correlate the data the methods previously employed to correlate bubble rise in clear liquids were reviewed. Because many of the bubbles were observed to be oblate spheroids, a new correlation was devised with the major diameter in the Reynolds number and the minor diameter in the drag coefficient.

\section{APPARATUS AND MEASUREMENTS}

The apparatus consisted of a glass cylinder containing the clear liquid to be used and a larger cylinder containing the packed material fully saturated with the liquid, Figure 1, Packing materials consisted of glass balls, 1 in. in diameter, packed in hexagonal, random, and simple cubic arrays, with porosities of 26.0 , 42.5 , and $47.6 \%$ respectively. The bubbles were introduced by means of a $1 / 4$ ml. hypodermic needle below the clear liquid cylinder. The bulk liquids were saturated with the bubble fluid to eliminate mass transfer. The rates of rise in the clear section as well as in the packed section were measured with a stop watch as the bubble rose continuously through both sections.
The liquids used were water-glycerine solution (70.6 wt.\% glycerine, viscosity 20.1 centipoise, $1.18 \mathrm{~g} . / \mathrm{ml}$.), tap water, and $n$-heptane. Temperatures were $23^{\circ}$ to $25^{\circ} \mathrm{C}$. Air was used as a bubble material for all three liquids. In addition benzene dyed red was used as droplets rising in the glycerine solution to obtain data at low Reynolds numbers. Those data in which the time of rise of the bubble was less than $0.8 \mathrm{sec}$. were eliminated because of the $0.1 \mathrm{sec}$. reaction time required to actuate the stop watch. Independent measurements of the bubble diameter vs. rise velocity were made on the same clear liquid systems as used in the packed beds employing the techniques described by Hartunian and Sears (I1). All liquids were U.S.P. grade, and al glassware was thoroughly cleaned with dichromate solution before use. Data were obtained on a range of bubble sizes from 0.1 to $0.01 \mathrm{in}$. diameter corresponding to Reynolds numbers from 0.1 to 1,000 . The screen supporting the packing passed all bubbles freely.

To correlate the data information available on bubble rise in clear liquids was

\section{PREVIOUS WORK ON BUBBLE RISE IN CLEAR LIQUIDS}

For clear liquids with bubbles rising in laminar flow the formulas of Stokes and Hadamard provide upper and lower bounds for the data (8). The Boussinesq theory gives the range of Reynolds numbers over which the reviewed. transition from Stokes' to Hadamard's law occurs (3). The Hadamard theory shows that internal circulation and reduced drag are associated with the equality of velocity in each fluid phase at the interface (9). The Boussinesq theory postulates a coefficient of surface viscosity $\epsilon$, not predictable theoretically, which implies secondary tangential stresses near the interface. For intermediate bubble velocities, with Reynolds' numbers from about 1 to 500 , Moore (17) has recently proposed relations which take into account inertial effects and which seek to explain the deformation of the bubbles into oblate spheroids. The quantitative results of these investigations are presented in Table 1 , where the drag coefficient is defined by

$$
C_{D}=\frac{4 g D_{0}}{3 u^{2}}\left(\frac{\rho_{L}-\rho_{g}}{\rho_{L}}\right)=\frac{a}{N_{R e}}
$$

Arnold and Millikan (1) obtained experimental data on the rise of bubbles in liquids and investigated the film-forming properties of fluids and their effect on drag coefficients. In the present investigation their data for air bubbles rising in olive oil were correlated with the arbitrarily chosen value of $\epsilon$ equal to $1.5 \times 10^{-4} \mathrm{lb}$.-mass $/ \mathrm{sec}$. in the Boussinesq relation of Table 1. The resulting curve passed through the experimental data and approached the Stokes and Hadamard curves at Reynolds numbers of about $10^{-4}$ and 1 respectively.

At a certain critical Reynolds number, varying with the liquid, the bubble shape oscillates and the upward path becomes either planar, zig-zag, or helical. Hartunian and Sears (11) investigated the unstable helical flow phenomenon and established a critical 


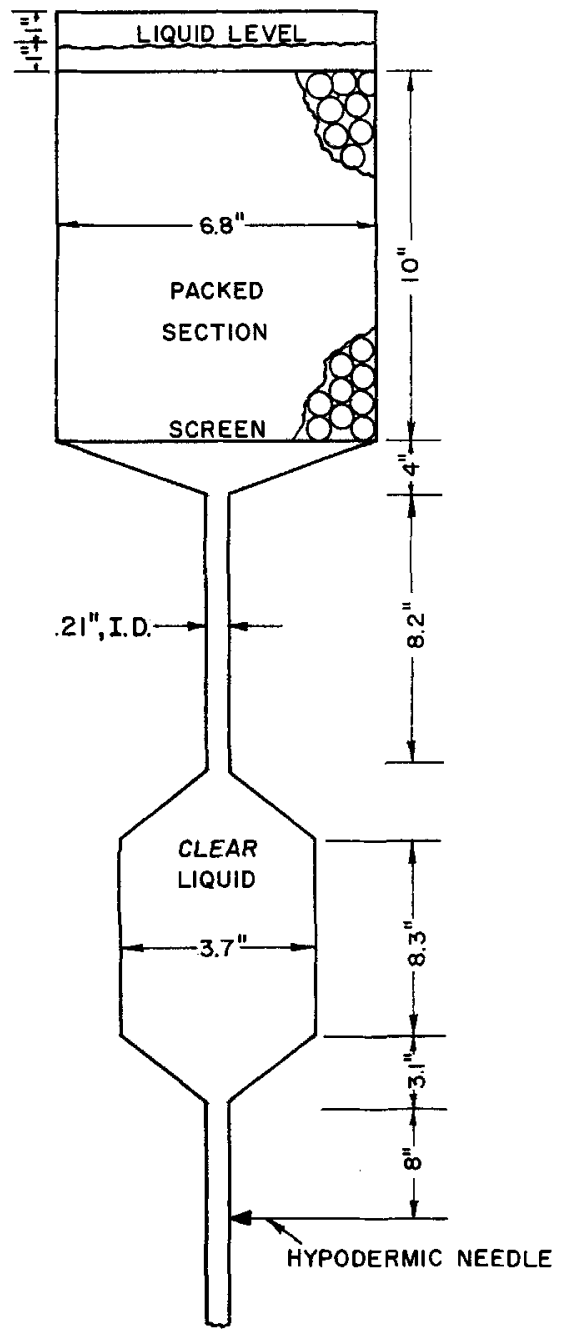

Fig. 1. Diagram of apparatus.

Reynolds number-Weber number curve for bubble stability.

At still higher $N_{R \theta}$ the bubbles are highly oblate and rise rectilinearly with a rocking motion about the vertical axis.

Finally for $N_{R s}$ greater than about 1,000 the shape is that of a spherical segment, and the bubble rises rectilinearly (spherical cap bubble). Davis and Taylor (5) presented the treatment of the rise of spherical cap bubbles in clear liquids.

A schematic diagram showing approximately the various modes of bubble rise is given in Figure 2. When drag coefficients are plotted vs. Reyn-

TABLE 1

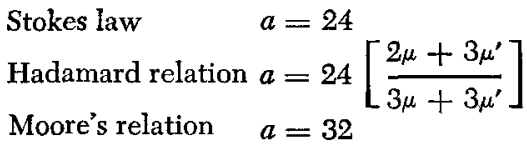

Boussinesq relation

$$
a=24\left[\frac{\epsilon+\left(2 \mu+3 \mu^{\prime}\right) r}{\epsilon+\left(3 \mu+3 \mu^{\prime}\right) r}\right]
$$

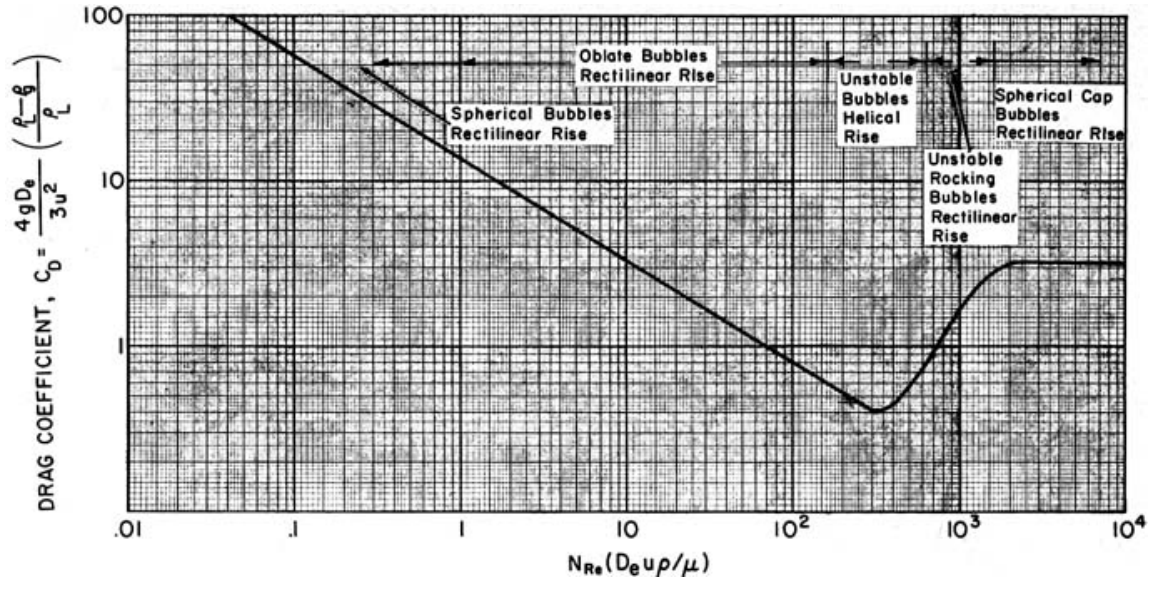

Fig. 2. Various modes of bubble rise in clear liquids.

olds number for many fluid-fluid systems, as in $(8)$, the spread of the data from a single curve is due to the following factors: degree of internal circulation (Boussinesq effect), variation of bubble shape related to Weber number, formation of films on bubble surface due to physicochemical reactions, and collection of small sized solid impurities at the fluid-fluid interface.

Further discussions of the physical behavior are to be found in the literature $(5,7,10,11,17,20)$. Special reference must be made to the bibliographies presented in $(10,11$, and 23) and to the theoretical developments in $(2,4,18,20$, and 21). Experimental data and descriptive material are presented in $(6,7,13,16,19,22,24$, and 25).

In this investigation the bubbles were observed to be spherical when small and deformed to oblate spheroids when larger.

\section{RECORRELATION OF DATA ON BUBBLE RISE IN CLEAR LIQUIDS}

A collection of experimental data on the rise of air bubbles in pure liquids has been made by Haberman and Morton (8). They correlated drag coefficient and Reynolds number on the basis of an equivalent diameter $D_{\text {e }}$. The data scatter considerably for different liquids. They photographed the rising bubbles and showed them to be variable in shape, as previously discussed.

A review of the development of the Reynolds number-drag coefficient relationships indicates that the question of the proper diameter to be used in each naturally arises.

The classical expression for the force balance on a gas bubble is (I4)

$$
\frac{\partial u}{\partial t}=\mathrm{g} \frac{\left(\rho_{L}-\rho_{g}\right)}{\rho_{g}}-\frac{C_{D} u^{2} \rho_{L} A_{p}}{2 m}
$$

The expressions for the mass and pro-

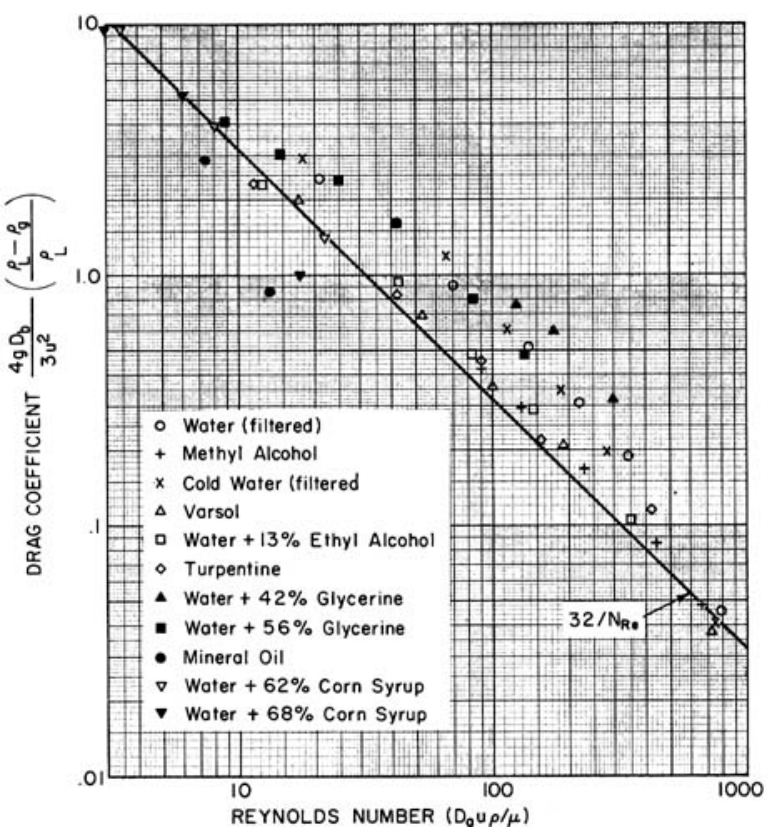

Fig. 3. Air bubbles rising in various liquids. 


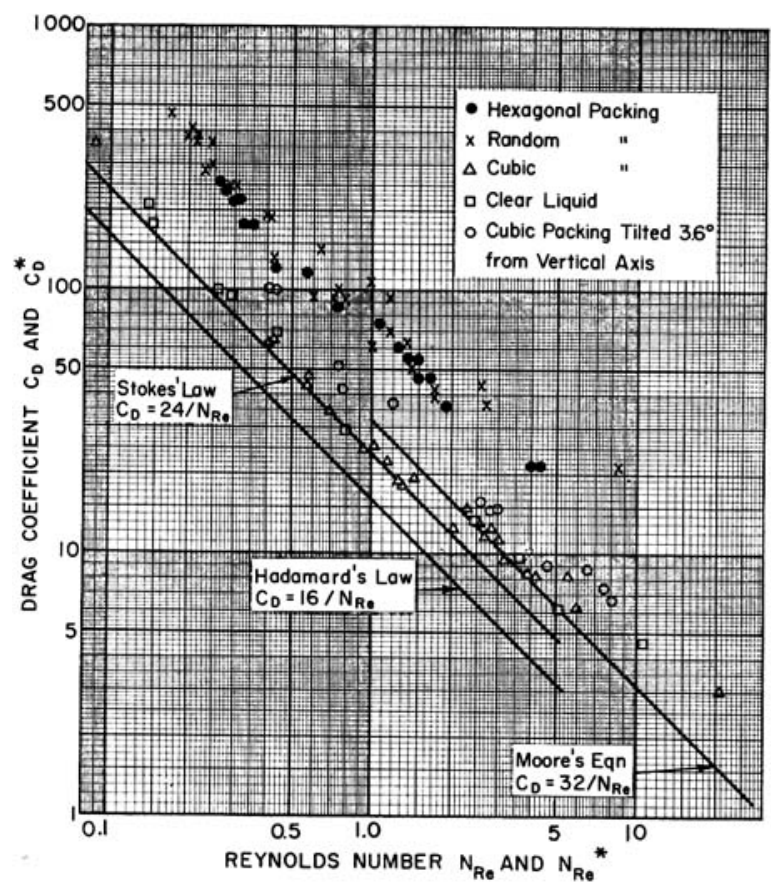

Fig. 4. Benzene droplets rising in glycerine solution.

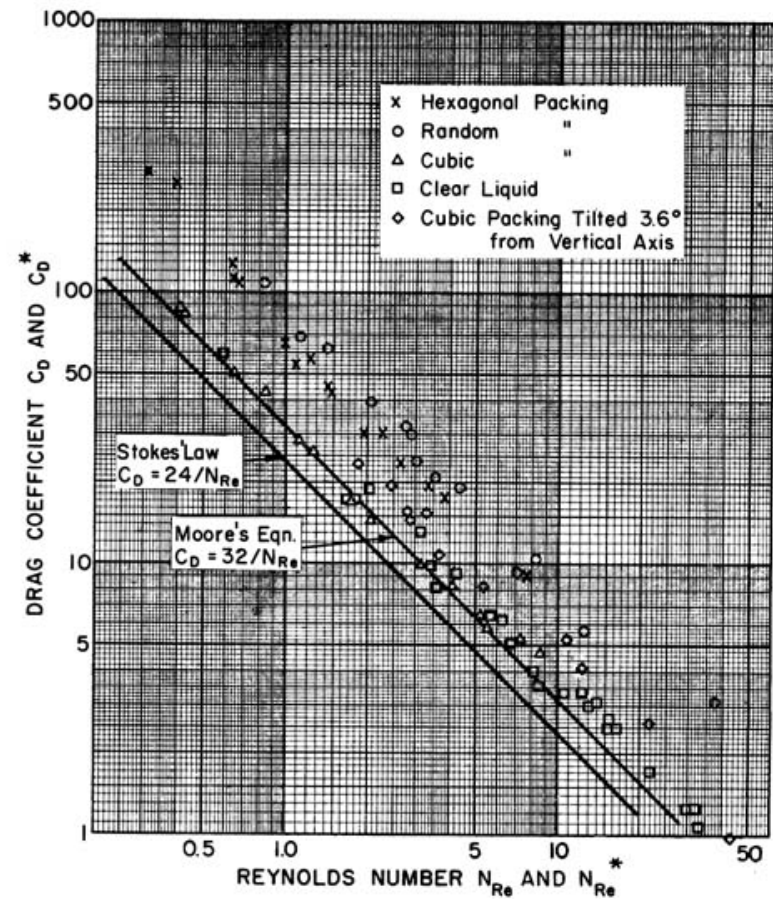

Fig. 5. Air bubbles rising in glycerine solution. jected area of an oblate spheroid are

$$
m=\frac{\pi D_{a}^{2} D_{b} \rho_{a}}{6} ; A p=\frac{\pi D_{a}{ }^{2}}{4}
$$

Introducing these relations into Equation (2) yields, for the steady state

$$
C_{D}=\frac{4 D_{o} g\left(\rho_{L}-\rho_{g}\right)}{3 u^{2} \rho_{L}}
$$

For the Reynolds number the precedent has always been to define the $N_{k e}$ in terms of some characteristic length in a plane perpendicular to the flow direction (14). In the case of oblate bubbles the preferable choice is the major diameter $D_{a}$. It is thus suggested that the $C_{D}$ and $N_{R o}$ be expressed in terms of the minor and major axes respectively. While it is true that $C_{D}$ will be very small for thin disks, bubbles become unstable before reaching this extreme shape.

The Weber number (with $D_{\theta}$ ) for bubbles with known volume and velocity are used to obtain the ratio of the minor diameter $\left(D_{a}\right)$ to the equivalent diameter $\left(D_{e}\right)$ by means of the relationship presented in Figure 7 of the paper by Hartunian and Sears (11). This ratio $\left(D_{a} / D_{s}\right)$, the volume of the bubble, and the formula for the volume of an oblate spheroid $(4 / 3 \pi$ $D_{a}{ }^{2} D_{b}$ ) permit the calculation of the major diameter $\left(D_{a}\right)$ and the minor diameter $\left(D_{b}\right)$.

In order to test the hypothesis outlined above the data of Haberman and Morton were recorrelated as outlined above. Points were selected from the smooth curves of velocity vs. equivalent diameter. From this information and the properties of the fluids the major and minor diameters were computed by the above procedure. The recomputed drag coefficients $\left(C_{D}\right)$ and Reynolds numbers $N_{k e}$ are plotted on Figure 3. It was found that for Reynolds numbers below about 25 there was little or no improvement in the correlation because the bubbles remained essentially spherical. As the bubble size increased and the shape tended toward spheroids, there was a significant decrease in the spread between the minimum and maximum drag coefficients at a given Reynolds number.
In the Reynolds number range of 100 to 500 the new correlation reduced the ratio of $C_{D}$ maximum $/ C_{D}$ minimum (that is spread of data) by factors of as much as 2.7 .

\section{CORRELATION OF NEW DATA}

It was decided that the new correlation just described would be used for the data taken in this research. Experimental data for the four systems are presented on Figures 4 to 7 . All the data in Figures 4 to 7 are confined to rectilinearly rising bubbles. Com-

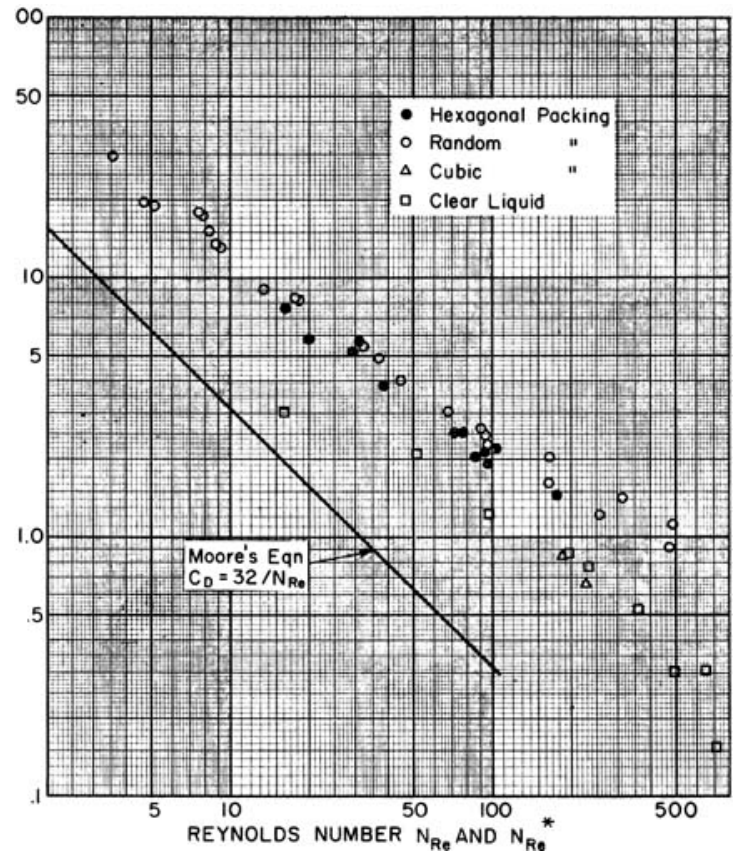

Fig. 6. Air bubbles rising in water. 
plete tabular data are available." Both the data for clear liquids and for packed beds are given on the same figure. In the packed bed the velocity is the integrated average velocity from the bottom to the top of the bed.

Although the effect of the packings is to reduce the velocity of the rising bubble, the degree of interference of the various arrangements would not necessarily be predicted from general considerations. For example the hexagonal and random packing give essentially the same drag, while the cubic array gives drag coefficients very similar to those in clear liquids because of the continuous vertical channel. The tilted cubic arrangement gives data somewhat in between cubic and random packing.

The data do not appear to be extensive enough with regard to packing arrangements and size of packing to warrant an evaluation of the parameters for the different packings. The random nature of the flow and the possible surface interactions give rise to a formidable analytical problem.

For random and hexagonal packings the bubble would bump against the lower surface of the glass sphere and come almost to a complete stop. The degree of slowing down of the bubble depended upon the obliqueness with which it contacted the lower surface of the 1-in. glass spheres. Bubbles striking exactly normal to the extreme lower surface seemed to stop completely. After reaching a minimum velocity the droplet accelerated very slowly along the curvilinear path defined by the sphere surface. This process was repeated over and over as the bubble traversed the bed. For the cubic packing the bubbles went up the center of the channel without touching the glass spheres. For the tilted cubic packing the bubbles were observed to move along the spherical surface without appreciable velocity change.

\section{CONCLUSIONS}

1. The drag coefficients for bubbles rising in hexagonal and random packed beds are two to three times those in clear liquids, while in cubic arrangements there is no significant difference.

2. The use of the minor and major diameters of the bubbles in the drag coefficient and Reynolds number gives an improved correlation for the rise of bubbles in clear liquids. The data, in this correlation, fall above the relationship derived by Moore (17). - Tabular material has been deposited as docuInstitute, Photoduplication Service, Library of Congress, Washington 25, D.C., and may be obtained for $\$ 1.25$ for photoprints or for $35-\mathrm{mm}$ microfilm.

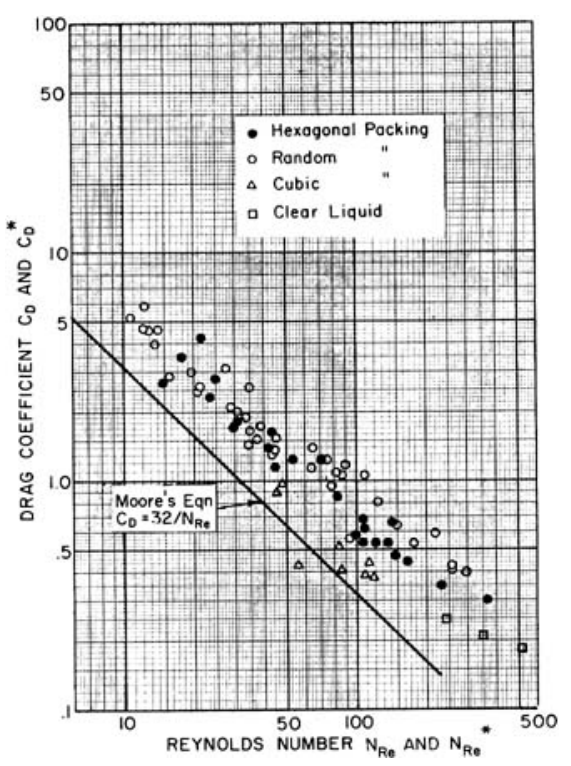

Fig. 7. Air bubbles rising in normal heptane.

3. The theory of Boussinesq (3) provides a model for correlating the bubble rise data in the very low Reynolds number range. Except for one arbitrary constant the theory predicts exactly the transition from the Stokes to the Hadamard regimes for the system examined.

\section{NOTATION}

$A_{p} \quad=$ projected area of bubble in flow direction (sq. ft.)

$C_{D} \quad=$ drag coefficient in clear liquid, based on $D_{b}, u$ Equation (1)

$C_{D}^{*}=$ drag coefficient in packed bed, based on $D_{b}^{*}, u^{*}$

$D_{e} \quad=$ equivalent diameter (of sphere of same volume as bubble) (ft.)

$D_{a} \quad=$ major diameter of spheroid in clear liquid (ft.)

$D_{a}^{*} \quad=$ major diameter of spheroid in packed bed, based on $u^{*}$ (ft.)

$D_{b}=$ minor diameter in clear liquid; $D_{b}^{*}$ in packed bed (ft.)

$\epsilon=$ coefficient of surface viscosity in Equation (5) (lb. mass/sec.)

g = acceleration of gravity (ft.) sec. ${ }^{2}$ )

$m=$ mass of bubble

$\mu \quad=$ viscosity of liquid; $\mu^{\prime}$ viscosity of bubble (lb.-mass/ ft. sec.)

$\rho_{L} \quad=$ density of ambient liquid; $\rho_{y}$ density of bubble or droplet (lb.-mass/cu. ft.)

$N_{R e}=$ Reynolds number in clear liquid based on $D_{a}, u$

$N_{R_{e}}{ }^{*}=$ Reynolds number in packed bed, based on $u^{*}, D_{a}^{*}$

$=$ radius of bubble

= surface tension, (lb.-force/ $\mathrm{ft}$. $u=$ velocity of bubble in clear liquid (ft./sec.)

$u^{*} \quad=$ velocity of bubble in packed bed (ft./sec.)

$N_{W e}=$ Weber number in clear liquid $u^{2} \times\left(\frac{\rho D_{\theta}}{g_{c}}\right)$

$N_{W_{0}}^{*}=$ Weber number in packed bed based on $u^{*}$

bubble includes both gas bubbles in liquids and drops of lower density immiscible liquid rising in more dense liquid

\section{LITERATURE CITED}

1. Arnold, H. D., Phil. Mag., 22, 755 (1911).

2. Bond, W. N., and D. A. Newton, ibid., 5, 794 (1928).

3. Boussinesq, J., Compt. Rend., 156, 1124 (1913).

4. Conkie, W. R, and P. Savic, Nat. Res. Counc., Canada, M. E. Rept., M.T. -23 (1953).

5. Davies, R. M., and G. I. Taylor, Proc. Roy. Soc. (London), A.200, 375 (1950).

6. Garner, F. H., and A. H. Skelland, Chem. Eng. Sci., 4, 149 (1955).

7. Nature, 173, 1239 (1954)

8. Haberman, W. L., and R. K. Morton, Proc. Am. Soc. Civil Engrs., 80, No. 387 (1954).

9. Hadamard, J., Compt. Rend., 152, 1735 (1911); 154, 107 (1912).

10. Hamarthy, T. Z., A.I.Ch.E. Journal, 6, 281 (1960).

11. Hartunian, R. A., and W. R. Sears, J. Fluid Mech., 3, 27 (1957).

12. Hughes, R. R., and E. R. Gilliland, Chem. Eng. Progr., 48, 493 (1952).

13. Ingebo, R., Natl. Advisory Comm. Aeronaut. Tech. Note 3773 (Sept., 1956).

14. Knudsen, J. G., and D. L. Katz, "Fluid Dynamics and Heat Transfer," McGraw-Hill, New York (1958).

15. McCabe, W. L., and J. C. Smith, "Unit Operations of Chemical Engineering," p. 356, McGraw-Hill, New York (1956).

16. McDonald, I., Birm. U. Chem. Engr., 10, 34 (1959).

17. Moore, D. W., J. Fluid Mech., 6, 113 (1959).

18. Pearcy, T., Austr. J. Physics., 9, 19 (1956).

19. Rosenberg, B., David Taylor Model Basin, Rept. 727 (1950).

20. Saffman, P., J. Fluid Mech., 1, 249 (1956).

21. Scriven, L. E., Chem. Eng. Sci, 12, 98 (1960).

22. Suckling, R., Birm. U. Chem. Engr., 11, 28 (1960).

23. Torobin, L., Can. J. Chem. Eng., 37, 129, I67, 224 (1959).

24. Uno, S., and R. C. Kintner, A.I.Ch.E. Journal, 2, 420 (1956).

25. Warshay, M., E. Bogusz, M. Johnson, and R. C. Kintner, Can. J. Chem. Eng., 37, 29 (1959).

Manuscript received October 28, 1960; revision received June 29,1961 ; paper accepted
July 5,1961 . 